\title{
Changing of the guard: reducing infection when replacing neural pacemakers
}

\author{
Joshua Pepper, MBBS, ${ }^{1}$ Lara Meliak, MD, ${ }^{1}$ Harith Akram, FRCS, ${ }^{1}$ Jonathan Hyam, DPhil, ${ }^{1,2}$ \\ Catherine Milabo, BSN, RGN, ${ }^{1}$ Joseph Candelario, BSc, ${ }^{1}$ Thomas Foltynie, PhD, ${ }^{1}$ \\ Patricia Limousin, PhD, ${ }^{1}$ Carmel Curtis, PhD, FRCPath, ${ }^{3}$ Marwan Hariz, PhD, ${ }^{1,4}$ and \\ Ludvic Zrinzo, PhD',2
}

\begin{abstract}
1 Unit of Functional Neurosurgery, University College London Institute of Neurology, Queen Square, London; ${ }^{2}$ Victor Horsley Department of Neurosurgery, National Hospital for Neurology and Neurosurgery, Queen Square, London; ${ }^{3}$ Department of Clinical Microbiology, University College London Hospital, London, United Kingdom; and ${ }^{4}$ Department of Clinical Neuroscience, Umeå University, Umeå, Sweden
\end{abstract}

\begin{abstract}
OBJECTIVE Infection of deep brain stimulation (DBS) hardware has a significant impact on patient morbidity. Previous experience suggests that infection rates appear to be higher after implantable pulse generator (IPG) replacement surgery than after the de novo DBS procedure. In this study the authors examine the effect of a change in practice during DBS IPG replacements at their institution.
\end{abstract}

METHODS Starting in January 2012, patient screening for methicillin-resistant Staphylococcus aureus (MRSA) and, and where necessary, eradication was performed prior to elective DBS IPG change. Moreover, topical vancomycin was placed in the IPG pocket during surgery. The authors then prospectively examined the infection rate in patients undergoing DBS IPG replacement at their center over a 3-year period with at least 9 months of follow-up.

RESULTS The total incidence of infection in this prospective consecutive series of 101 IPG replacement procedures was $0 \%$, with a mean follow-up duration of $24 \pm 11$ months. This was significantly lower than the authors' previously published historical control group, prior to implementing the change in practice, where the infection rate for IPG replacement was $8.5 \%$ (8/94 procedures; $p=0.003)$.

CONCLUSIONS This study suggests that a change in clinical practice can significantly lower infection rates in patients undergoing DBS IPG replacement. These simple measures can minimize unnecessary surgery, loss of benefit from chronic stimulation, and costly hardware replacement, further improving the cost efficacy of DBS therapies.

https://thejns.org/doi/abs/10.3171/2016.4.JNS152934

KEY WORDS deep brain stimulation; implantable pulse generator; infection; Parkinson's disease; reoperation; antibiotic; vancomycin; functional neurosurgery

$\mathrm{D}$ EEP brain stimulation (DBS) was popularized by the Grenoble group ${ }^{2}$ and surpassed stereotactic ablation as the predominant treatment in functional neurosurgery at the end of the last millennium. DBS is now an established treatment for a number of movement disorders including Parkinson's disease, dystonia, and tremor. This has raised interest in the possible use of DBS for severe and unremitting psychiatric disorders including obsessive-compulsive disorder and depression. ${ }^{31}$

Despite the surge in popularity of DBS only a small number of publications have specifically analyzed the complications of DBS surgery, $3,4,6,15,32,40,41$ and even fewer have assessed interventions that can reduce the rate of adverse events. $3,14,24,26,28,37,46$ Reported infection rates vary between centers from $0 \%$ to $22 \%{ }^{7,42}$ Our group recently published data demonstrating that infection rates after implantable pulse generator (IPG) replacement are significantly higher when compared with other types of DBS-related surgery. ${ }^{32}$ Other authors have reported similar trends. ${ }^{3}$ However, the overall picture is far from clear, as other groups reported infection rates similar to de novo surgery. ${ }^{4,36}$

Miller and colleagues previously reported a significant improvement in hardware-related infection in all stereotactic and functional neurosurgical procedures with

ABBREVIATIONS DBS = deep brain stimulation; IPG = implantable pulse generator; MRSA = methicillin-resistant Staphylococcus aureus.

SUBMITTED December 22, 2015. ACCEPTED April 7, 2016.

INCLUDE WHEN CITING Published online June 17, 2016; DOI: 10.3171/2016.4.JNS152934. 
the use of topical antibiotics. ${ }^{28}$ Moreover, it is well documented that colonization with Staphylococcus aureus is an independent risk factor for the development of postsurgical infection, ${ }^{44}$ and this risk may be as high as 33\% ${ }^{29}$ Therefore, since January 2012 we adapted our surgical procedure with the aim of reducing the rate of infection after IPG replacement surgery. Together with our local infection control team we introduced the use of methicillinresistant $S$. aureus (MRSA) screening and eradication as well as an intraoperative topical vancomycin wash during IPG replacement. The aim of this prospective study was to assess whether this change in practice made a significant impact on infection rates after IPG replacement surgery when compared with historical controls.

\section{Methods}

Surgical reports and clinical notes were reviewed in all 171 patients ( 89 men and 82 women) who underwent IPG replacement surgery at the National Hospital of Neurology and Neurosurgery, Queen Square, London, from November 2002 until December 2014. Prior to January 2012 all data collected were retrospective. From January 2012 until December 2014 all patients undergoing IPG replacement surgery were prospectively followed up.

Baseline patient characteristics (age at surgery, sex, diagnosis, brain target) as well as details of the operation performed were collected for each patient. Before January 1 , 2012, the IPG pocket was vigorously washed with copious saline. After this date all patients underwent MRSA screening and eradication where appropriate prior to surgery, and at surgery the IPG pocket was instilled with a vancomycin wash prior to closure (further details below). Patients were grouped into a historical "control" group and a "prospective" group following this change in practice. All patients had a minimum follow-up duration of 9 months.

Information on any DBS-related infection, including type, site, and microbiological diagnosis, was collected for all patients with infection. The definition of recorded infection is the same as in our previous study. ${ }^{32}$ Only infections in direct relation to the hardware were considered. Infections were defined if any of the following were present: 1) clinical suspicion of an infection (i.e., redness, swelling, warmth, or fluid surrounding any of the DBS components beyond that expected due to postsurgical inflammation, with either elevated temperature or inflammatory markers); 2) purulent exudates from the suspected site of infection; 3) microbiological evidence; and 4) skin erosion with any of the above. The infection rate was calculated as the number of infections per patient as well as the number of infections per procedure.

\section{Microbial Screening and Eradication Protocol}

All patients in the prospective group attended a preoperative assessment clinic where they underwent skin and nasal screening for MRSA colonization. All patients who had negative colonization results continued to surgery without eradication. Patients underwent MRSA eradication if they 1) had a positive MRSA colonization result, 2) were unable to attend preoperative assessment clinic, 3) were historically MRSA-colonization positive, or 4) were a known MRSA carrier undergoing delayed elective surgery with or without previous eradication. All patients with delayed elective surgery underwent repeat MRSA screening (Fig. 1). Table 1 lists details of the eradication protocol.

\section{Replacement of IPG}

Patients received a single dose of $1.5 \mathrm{~g}$ of intravenous cefuroxime or a single dose of $500 \mathrm{mg}$ of intravenous clarithromycin in cases of penicillin allergy at induction. If patients were MRSA-positive, $6 \mathrm{mg} / \mathrm{kg}$ of intravenous teicoplanin was added to the primary prophylaxis. With the patient under general or local anesthesia, the pocket of the IPG was opened via the old scar. If the scar was unsightly, the scar was excised together with an ellipse of skin. The old IPG was replaced with the new IPG placed in the fibrous pocket. When exchanging the old IPG for more bulky hardware (such as a Kinetra IPG being replaced with an Activa PC via a cable adapter [Medtronic]), the new hardware was sometimes placed in a deeper pocket, formed beneath the pectoralis major muscle via a musclesplitting approach, especially when the overlying skin was deemed thin.

Before January 1, 2012, the pocket was washed with copious amounts of saline. After this date, a vancomycin/saline wash was used $(20 \mathrm{ml}$ of $1 \mathrm{mg} / \mathrm{ml}$ vancomycin solution). The wound was closed in layers with carefully buried absorbable sutures and interrupted nylon for skin closure. The patient received 3 further doses of $750 \mathrm{mg}$ of intravenous cefuroxime at 8-hour intervals or the appropriate alternative if the patient was allergic to penicillin or positive for MRSA. Dressings were not removed unless they were heavily blood stained, and sutures were removed after 10-14 days. Patients were instructed to keep the wound dry until 24 hours after suture removal.

\section{Statistical Analysis}

An unpaired Student t-test was used to compare the ages of patients with and without infection, between groups, and the number of surgeons per operation. The infection rate between groups was compared with a Fisher exact test using a $2 \times 2$ contingency table. A p value $<0.05$ was considered significant.

\section{Results}

\section{Baseline Characteristics}

One hundred seventy-one patients underwent a total of 195 IPG replacement procedures. Of those in the historical control group, 80 patients underwent 94 IPG replacement surgeries (mean age $48 \pm 20$ years old, $48 \%$ male). This included 15 procedures (16\%) in which patients had 1 or more previous IPG replacement surgeries. In the "prospective" group, 91 patients underwent 101 IPG replacement surgeries (mean age $54 \pm 15$ years, 56\% male) and included 24 procedures $(23.8 \%)$ in which patients had 1 or more previous IPG replacement surgeries. There was no significant difference in sex distribution, primary indication for DBS surgery, brain target, or rates of diabetes between the 2 groups (Table 2).

Minimum follow-up duration was 9 months in both groups, with a mean follow-up duration of $24 \pm 11$ months 


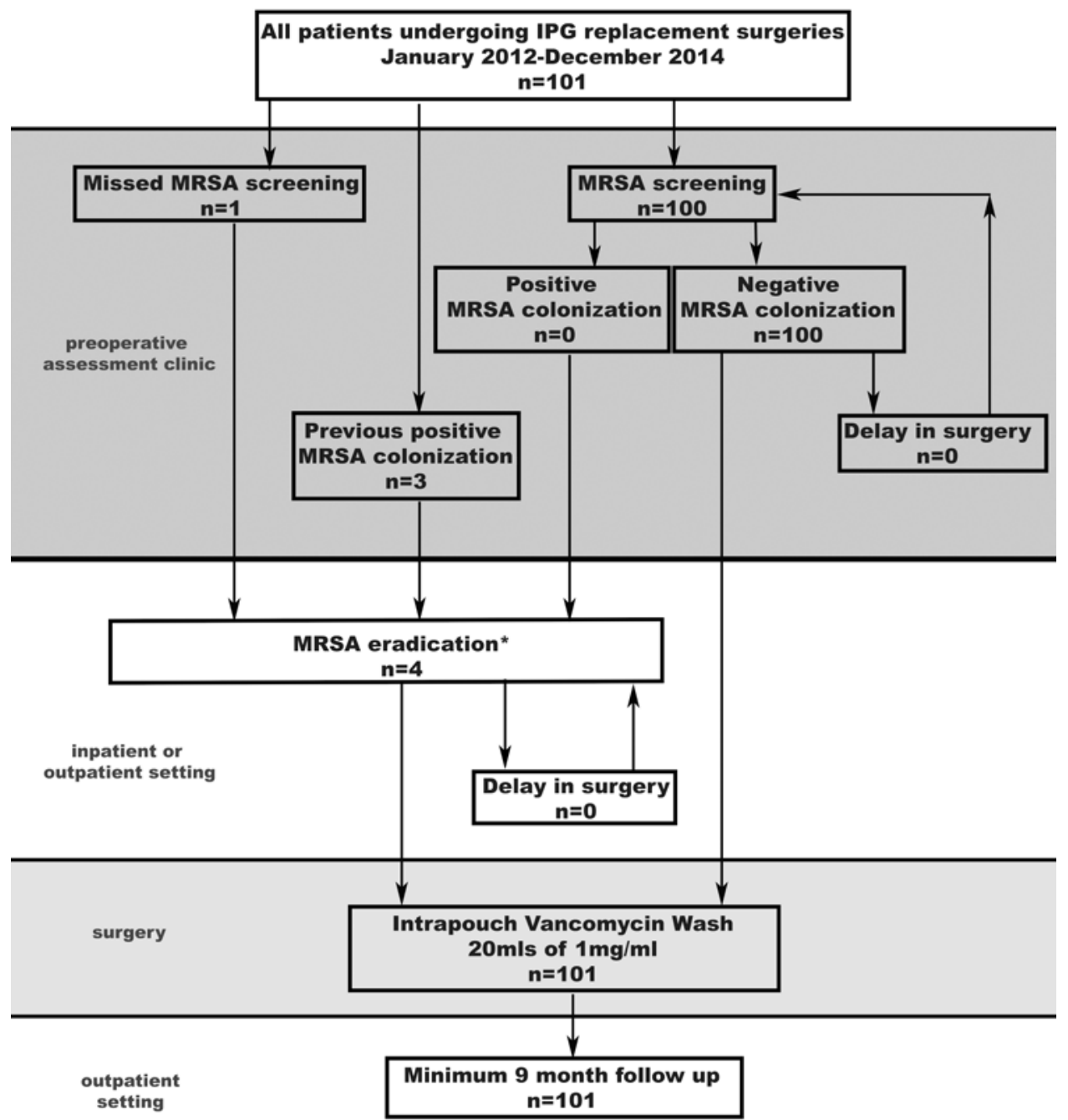

FIG. 1. Flow chart showing the new IPG replacement protocol. * See Table 1 for the MRSA eradication protocol.

in the prospective group and $73 \pm 26$ months in the control group ( $p=0.0001$ ). The number of surgeons involved was higher in the control than in the prospective group (1.8 vs 1.4 surgeons, respectively; $p=0.0002$ ). Patients in the prospective group were older, had a smaller proportion of patients with dystonia and Parkinson's disease, and had undergone more previous IPG changes (Table 2).

\section{Rate of MRSA Colonization in the Prospective Cohort}

There were no confirmed MRSA colonizations within the prospective group. However, 4 patients underwent MRSA eradication: 3 who had historical MRSA colonization and 1 who failed to attend the MRSA screening (Fig. 1).

\section{Postoperative Infections}

In total, 8 postoperative infections occurred in 6 patients in the historical control group. This corresponds to a patient infection rate of $7.5 \%$ and a procedure infection rate of $8.5 \%$. There were no infections in the prospective group, and this difference was statistically significant ( $p$
$=0.003$; Fig. 2). Information on patients with infection is detailed in brief in Table 3 and in more detail in our previous publication. ${ }^{32}$ The mean duration until infection in the control group was $3.1 \pm 5.8$ months. None of the infections were secondary to MRSA.

\section{Reason for IPG Replacement}

The most common reason for IPG replacement was depletion/near depletion of the IPG's battery in both groups. Replacement of the IPG after prior hardware removal because of infection was the indication in $12 \%$ of the historical control group compared with $0 \%$ in the prospective group $(\mathrm{p}=0.0002$; Table 4).

\section{Adverse Events}

There were no adverse events noted in relation to the use of topical vancomycin wash or microbial screening/ eradication.

\section{Patients With Previous DBS-Related Infections}

The historical control group contained 1 patient (1/80, 
TABLE 1. MRSA eradication protocol during IPG replacement*

\begin{tabular}{|c|c|c|c|}
\hline $\begin{array}{l}\text { Type of } \\
\text { Wash }\end{array}$ & Antimicrobial & Frequency & $\begin{array}{l}\text { Length } \\
\text { of Time } \\
\text { (days) }\end{array}$ \\
\hline Body wash & Chlorhexadine gluconate $4 \% \dagger$ & Once per day & 6 \\
\hline Hair wash & Chlorhexadine gluconate $4 \% \dagger$ & Every other day & 5 \\
\hline Nasal & Mupirocin $2 \%$ & 3 times per day & 5 \\
\hline \multicolumn{4}{|c|}{$\begin{array}{l}\text { * Protocol schedule as follows: Day 1, body wash, hair wash, and nasal; Day } \\
2 \text {, body wash and nasal; Day 3, body wash, hair wash, and nasal; Day 4, body } \\
\text { wash and nasal; Day 5, body wash, hair wash, and nasal; and Surgery, body } \\
\text { wash. } \\
\dagger \text { Octenisan (Schülke) was used as a substitute if chlorhexadine gluconate } 4 \% \\
\text { was contraindicated. }\end{array}$} \\
\hline
\end{tabular}

$1.2 \%$ ) with a previous DBS-related infection, and the prospective cohort contained 4 patients $(4 / 91,4.4 \%)$ who had previous infection of DBS hardware. This difference was not statistically significant.

\section{Special Cases}

Of the 6 patients with IPG infections in the historical group, 2 deserve further discussion. Patient 2 underwent surgery for tardive dystonia and suffered 3 infections after IPG replacement surgery and 4 in total. The initial infection affected the IPG alone and was managed by cutting of the cables below the cranial connector site and extraction of the cut cables and IPG. The subsequent infection occurred 2 months later and was associated with erosion of the distal end of the cut extension cable through the skin, and the cable stump was removed. Four weeks later a purulent spot over the left pericoronal wound was noted, and the patient was admitted to the hospital for removal of the leads. These were all considered separate infections as they occurred at different time points all related to the most recent surgery (IPG replacement). Three and a half months later the entire system was reimplanted, but the patient developed a subcutaneous infection around the IPG pocket that resulted in removal of the infected components, leaving the leads in place. Finally, new cables and an IPG were reinserted 4 months later. This patient suffered from Type II diabetes mellitus.

Patient 3 with severe dystonia and cachexia underwent IPG replacement due to near depletion of the battery and was discharged back to his nursing home. The patient presented to the hospital 17 months later with erosion of the IPG through the skin. On presentation no clinical signs of infection were present and an attempt was made to rescue the IPG by excising the wound margins and forming a new pocket. This was followed by development of a purulent infection.

\section{Discussion}

The use of an intraoperative vancomycin wash and microbial screening significantly reduced the rate of infection after IPG replacement surgery from a procedure infection rate of $8.5 \%$, and patient infection rate of $7.5 \%$, to $0 \%$.

In 5 of 6 patients with infection in the historical control group, the causative organism of purulent infection was
TABLE 2. Baseline characteristics

\begin{tabular}{lccc}
\hline \multicolumn{1}{c}{ Variable } & Control & Prospective & p Value \\
\hline No. of patients & 80 & 91 & - \\
\hline No. of procedures & 94 & 101 & - \\
\hline Mean age (SD) & $48(20)$ & $54(15)$ & $0.018^{*}$ \\
\hline Male (\%) & 48 & 56 & 0.25 \\
\hline Mean follow-up in mos (SD) & $73(26)$ & $24(11)$ & $0.0001^{*}$ \\
\hline Diabetes mellitus \% (no.) & $1(1 / 80)$ & $2(2 / 91)$ & 0.80 \\
\hline Mean no. of surgeons (SD) & $1.8(0.7)$ & $1.4(0.6)$ & $0.0002^{*}$ \\
\hline Parkinson's disease (\%) & 54 & 45 & 0.23 \\
\hline Dystonia (\%) & 42 & 42 & 1.0 \\
\hline Other diagnosis (\%) & 3 & 14 & $0.02^{*}$ \\
\hline Subthalamic nucleus (\%) & 48 & 37 & 0.17 \\
\hline Globus pallidus internus (\%) & 49 & 57 & 0.29 \\
\hline Other targets (\%) & 4 & 6 & 0.73 \\
\hline Patients w/ previous infections (\%) & 1.2 & 4.4 & 0.37 \\
\hline No. w/ $\geq 1$ previous IPG change & 15 & 24 & 0.58 \\
\hline No. w/ $\geq 2$ previous IPG changes & 4 & 15 & $0.048^{*}$ \\
\hline * Statistically significant. & & &
\end{tabular}

Staphylococcus species. The rate of skin colonization of S. aureus is more than $70 \%$ in patients with confirmed skin and soft-tissue infections. ${ }^{10,25}$ The most common causative organism responsible for hardware infection in DBS surgery is staphylococcal species. ${ }^{3,4,32}$ Importantly, despite proper skin disinfectant and draping during surgery, bacteria including coagulase-negative staphylococci and Propionibacterium acnes will begin to colonize the skin within 1 hour, with a marked bacterial colonization load at 2 hours. ${ }^{12}$

S. aureus is a common commensal species that affects up to two-thirds of healthy individuals throughout their lifetime. ${ }^{22,25}$ The skin provides a remarkably resistant barrier to infection by colonized bacteria due to a large extent on the production of antimicrobial proteins such as defensins and cathelicidins. ${ }^{30}$ Skin breaches during surgery allow the spread of $S$. aureus within the skin and deeper tissue layers. MRSA infections can be more serious than methicillin-sensitive $S$. aureus infections due to their reduced sensitivity to commonly used antibiotics and result in worse clinical outcomes. ${ }^{45}$

Within the National Health Service in England, Public Health England reports that the rate of MRSA bacteremia $^{16}$ and surgical site infections ${ }^{9}$ have fallen markedly over the last decade. The reason for this is unclear, but screening and subsequent isolation and eradication protocols are believed to be contributory. ${ }^{43}$

Topical application of antibiotics produces far higher intrawound concentrations than would be possible via intravenous administration alone. This is especially true in patients undergoing IPG replacement surgery where the subcutaneous fibrous pocket has a reduced blood supply. The topical use of a variety of antibiotics has long been used effectively in treatment and prophylaxis of wound infection. ${ }^{5,33,35}$ Moreover, in neurosurgical shunt operations the use of a local antibiotic injected directly into the shunt 


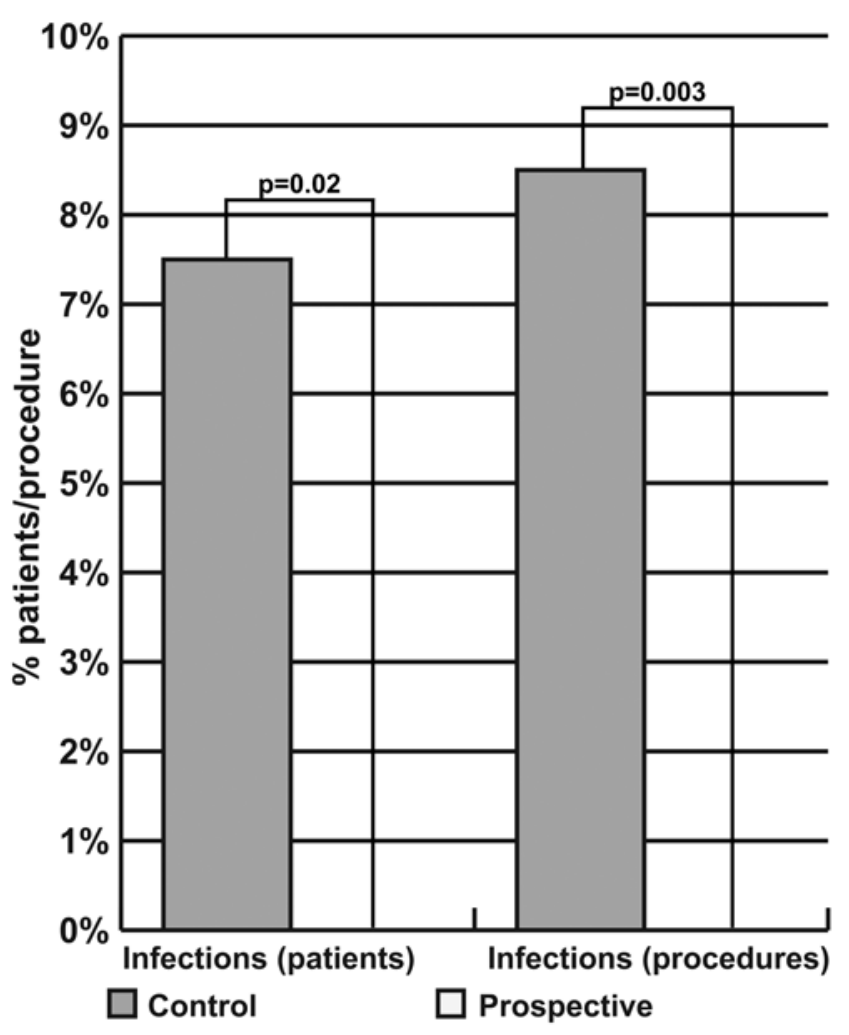

FIG. 2. Graph showing the percentage of patients and procedures with infections.

reduced the infection rate from $6 \%$ to $0.4 \%$ in a controlled trial of 802 shunt procedures. ${ }^{34}$

Staphylococcus species are highly sensitive to vancomycin. Thus, the use of a local vancomycin wash into subcutaneous tissue is judicious in hardware implantation surgery. In this study the use of a vancomycin wash in more than 100 procedures with an average follow-up duration of 2 years has resulted in no infections. Importantly, there were no adverse events noted from the use of vancomycin wash.

\section{Change in Antibiotic Regimen}

Bhatia and colleagues ${ }^{3}$ changed the perioperative an- tibiotic regimen from intravenous cefuroxime to intravenous vancomycin and gentamicin. This change reduced the overall infection rate and importantly reduced their rate of infection after IPG replacement surgery from $17.6 \%$ to $3.6 \%$. While highlighting an important trend, this study was underpowered, and the outcome was not statistically significant. The causative organism in this original report was Staphylococcus species in two-thirds of the cases of IPG replacement. In the highly detailed review conducted by the authors, the causative organism of infection was Staphylococcus species in more than 50\% of cases.

Miller et al..$^{28}$ meticulously collected information on all stereotactic and functional hardware procedures over a 5-year period. In total, 614 patients underwent a variety of procedures including DBS, spinal cord stimulator, peripheral nerve stimulator, and other device implantations. All patients were given perioperative cephalosporin or vancomycin. In the final 18 months of this study, all subcutaneous pockets were irrigated with neomycin/polymyxin as opposed to saline prior to skin closure. The overall rate of infection was reduced from $5.7 \%$ to $1.2 \%(\mathrm{p}<0.05)$ in the group with the antibiotic washout. The causative organism of infection was Staphylococcus species in $82 \%(23 / 28)$ of cases.

In other fields of surgery and neurosurgery involving implantable hardware the use of topical vancomycin has led to marked reductions in the rate of postoperative infections. The use of a topical vancomycin powder in instrumented spinal surgery in a prospectively followed cohort led to a reduction in postoperative infection rates from $12.5 \%$ to $0 \%,{ }^{23}$ a finding similar to our own.

\section{Microbial Screening and Eradication}

Colonization with $S$. aureus is an independent risk factor for the development of postoperative surgical site infections. ${ }^{21,44}$ In our study, information on MRSA carrier status was not available for all historical controls because routine screening was not conducted until January 2012. Moreover, MRSA was not cultured from any of our infected patients. Nevertheless, prior knowledge of MRSA status led to prophylactic decontamination in 3 patients within our prospective cohort. Although we suspect that

TABLE 3. Characteristics of patients in the historical control group who suffered infection following IPG replacement*

\begin{tabular}{|c|c|c|c|c|c|c|}
\hline $\begin{array}{l}\text { Case } \\
\text { No. }\end{array}$ & $\begin{array}{l}\text { Age } \\
\text { (yrs) }\end{array}$ & Diagnosis & $\begin{array}{l}\text { Time to } \\
\text { Infection }\end{array}$ & $\begin{array}{l}\text { Hardware } \\
\text { Involved }\end{array}$ & Type of Infection & Culture Result \\
\hline 1 & 61 & PD & 2 wks & IPG & Suppurative & S. aureus \\
\hline \multirow[t]{3}{*}{2} & 63 & Dystonia & 3 days & IPG & Suppurative & Coagulase-negative staphylococcus \\
\hline & 64 & Dystonia & $2 \mathrm{mos}$ & Cable & Erosion through skin & \\
\hline & 64 & Dystonia & 4 mos & Electrode (scalp) & Purulent spot around It frontal incision & \\
\hline 3 & 36 & Dystonia & $17 \mathrm{mos}$ & IPG & Erosion of IPG through skin & Candida albicans \\
\hline 4 & 40 & Dystonia & 9 days & IPG, cable & Suppurative & Electrode tip: S. epidermidis \\
\hline 5 & 43 & Dystonia & 3 days & Cable, electrode (scalp \& brain) & Suppurative, cerebral abscess & S. aureus \\
\hline 6 & 43 & Dystonia & $1 \mathrm{mo}$ & IPG & Suppurative & S. aureus \\
\hline
\end{tabular}

$\mathrm{PD}=$ Parkinson's disease.

* All patients were male. The target for DBS was the globus pallidus internus, bilaterally. Hardware was removed in all patients. 
TABLE 4. Reasons for IPG change

\begin{tabular}{lccl}
\hline \multicolumn{1}{c}{ Reason } & Control & Prospective & p Value \\
\hline Depleted battery & 80 & 93 & 0.17 \\
\hline Infection & 11 & 0 & 0.0002 \\
\hline Malfunction & 2 & 4 & 0.7 \\
\hline Discomfort & 1 & 2 & 1.0 \\
\hline Other $^{*}$ & 0 & $2^{*}$ & 0.5 \\
\hline
\end{tabular}

* Other includes 1 patient who required more complex programming and a second patient who had a poor response to a new IPG so an old model was reimplanted.

the majority of the benefit observed in terms of reduced infection rates was due to the use of topical vancomycin, we cannot discount a contribution from the introduction of routine MRSA screening.

\section{Diabetes Mellitus}

It is noted in neurosurgery ${ }^{11}$ and other fields of surgery ${ }^{29}$ that diabetes is an independent risk factor for the development of postoperative surgical infections. Importantly, in relation to this study, patients with MRSA colonization who underwent cardiac surgery were more likely to develop postoperative infection if they had a confirmed preoperative diagnosis of diabetes. ${ }^{21}$

In our patient groups, the rate of diagnosed diabetes mellitus was low and similar between the two groups. This rate is similar to other published series of DBS patients. ${ }^{36,41}$ Due to the low number of diagnoses, we cannot comment on its significance as a contributory factor in the development of postoperative infections. However, a number of other DBS-related publications have noted that diabetes does not appear to be correlated with the development of postoperative infections..$^{20,39,41}$

\section{Timing of Infection and Follow-Up}

In this study, 6 (75\%) of 8 IPG infections in the control group occurred within 2 months of surgery, $7(88 \%)$ of 8 occurred within 6 months, and all infections occurred within 17 months of surgery. The mean follow-up duration of 24 months and minimum follow-up of 9 months in the prospective group may thus be considered adequate to determine the rate of postprocedural infections. Therefore, it is unlikely that the (inevitably) shorter duration of followup in the second group contributed to the lower rate of infection in any meaningful way. Other studies support the notion that the majority of postsurgical infections (roughly $80 \%$ ) occur within the first few months of surgery with a smaller proportion, often not directly related to surgery, occurring at a later stage, usually up to 2 years after the procedure. . $^{3,4,32}$

\section{Number of Surgeons}

The number of surgeons was higher in the control group on average by 0.4 surgeons over 101 procedures. This might have contributed to the higher infection rate within the control group, although this is uncertain. Other groups have noted that the number of individuals present in the operating room is related to a higher infection rate. ${ }^{39}$
However, at our institution the consultant (attending) surgeon listed in the operative record and supervising the procedure does not always scrub for the procedure. Therefore, these numbers must be interpreted with caution, and it is not clear whether the number of scrubbed surgeons is a significant contributing factor.

\section{Number of Previous IPG Replacement Surgeries}

We postulated in our previous publication that the number of IPG replacement surgeries might be an independent risk factor for infection. ${ }^{32}$ Our reasoning was 2-fold: that the fibrous pocket around the IPG does not provide an adequate inflammatory response to infection, and the likely reduced penetrance of prophylactic intravenous antibiotics increases the likelihood of an infection. The number of pacemaker changes is a risk factor for infection when compared with de novo implantation in cardiac surgery. ${ }^{1,8,17,18}$ Furthermore, in hip replacement surgery the rate of infection is $18 \%$ higher after repeat revision when compared with de novo surgery. ${ }^{27}$ A recent publication has confirmed an increasing infection rate in multiple DBS IPG changes. ${ }^{38}$

In our prospective group, $15 \%$ of all surgeries occurred in patients who had 2 or more previous IPG replacements, and almost a quarter of all patients had at least 1 previous IPG change. This is to be compared with $4 \%(\mathrm{p}=0.048)$ and $16 \%(\mathrm{p}=$ nonsignificant $)$ of patients in our control group, respectively. Although the number of IPG changes is an independent risk factor for infection, our protocol appears to significantly negate this risk in IPG replacement surgery.

\section{Study Limitations}

Comparison of a prospective cohort with a historical control group may have produced potential confounding that contributed to the difference in infection rates. Nevertheless, our DBS service has always been alert to the importance of minimizing infection. No changes were made to the surgical procedure in the prospective group other than the addition of topical vancomycin wash. Surgical learning curves may also lead to higher early complication rates. ${ }^{13}$ However, a surgical learning curve may not be present when a relatively simple procedure is performed by an experienced surgeon. ${ }^{19}$ Indeed, the majority of infections in the historical control group did not occur in the early years of our DBS service. ${ }^{32}$ Moreover, the prospective group was older with more repeat IPG changes, both independent risk factors for infection. These factors suggests that a greater baseline risk for the prospective group was reversed by using topical vancomycin wash.

\section{Conclusions}

The use of topical vancomycin has significantly reduced the rate of infection after IPG replacement surgery. MRSA screening and eradication may also have contributed to the reduced infection rate. No adverse events were noted, and our protocol appears to have negated the increased risk of infection associated with multiple IPG changes. These simple measures prevented unnecessary surgery, loss of benefit from chronic stimulation, and costly hardware 
replacement, further improving the cost efficacy of DBS therapies.

\section{Acknowledgments}

This work was undertaken at University of College London (UCL) and UCL Hospitals, and was partly funded by the Department of Health National Institute for Health Research Biomedical Research Centres funding scheme. The Unit of Functional Neurosurgery, UCL Institute of Neurology, is supported by the Sainsbury Monument Trust and The Parkinson's Appeal for Deep Brain Stimulation.

\section{References}

1. Bloom H, Heeke B, Leon A, Mera F, Delurgio D, Beshai $\mathrm{J}$, et al: Renal insufficiency and the risk of infection from pacemaker or defibrillator surgery. Pacing Clin Electrophysiol 29:142-145, 2006

2. Benabid AL, Pollak P, Louveau A, Henry S, de Rougemont $\mathrm{J}$ : Combined (thalamotomy and stimulation) stereotactic surgery of the VIM thalamic nucleus for bilateral Parkinson disease. Appl Neurophysiol 50:344-346, 1987

3. Bhatia R, Dalton A, Richards M, Hopkins C, Aziz T, Nandi $\mathrm{D}$ : The incidence of deep brain stimulator hardware infection: the effect of change in antibiotic prophylaxis regimen and review of the literature. Br J Neurosurg 25:625-631, 2011

4. Bjerknes S, Skogseid IM, Sæhle T, Dietrichs E, Toft M: Surgical site infections after deep brain stimulation surgery: frequency, characteristics and management in a 10-year period. PLoS One 9:e105288, 2014

5. Lindsey D, Nava C, Marti M: Effectiveness of penicillin irrigation in control of infection in sutured lacerations. J Trauma 22:186-189, 1982

6. Blomstedt P, Hariz MI: Hardware-related complications of deep brain stimulation: a ten year experience. Acta Neurochir (Wien) 147:1061-1064, 2005

7. Chou YC, Lin SZ, Hsieh WA, Lin SH, Lee CC, Hsin YL, et al: Surgical and hardware complications in subthalamic nucleus deep brain stimulation. J Clin Neurosci 14:643-649, 2007

8. Connolly SJ, Gent M, Roberts RS, Dorian P, Roy D, Sheldon RS, et al: Canadian implantable defibrillator study (CIDS): a randomized trial of the implantable cardioverter defibrillator against amiodarone. Circulation 101:1297-1302, 2000

9. Elgohari S, Mihalkova M, Wloch C, Saei A, Harris R, Charlett A, et al: Surveillance of Surgical Site Infections in NHS Hospitals in England 2013/14. London: Public Health England, 2014 (https://www.gov.uk/government/ uploads/system/uploads/attachment_data/file/386927/SSI_ report_2013_14_final__3_.pdf) [Accessed 15 September, 2015]

10. Ellis MW, Schlett CD, Millar EV, Crawford KB, Cui T, Lanier JB, et al: Prevalence of nasal colonization and strain concordance in patients with community-associated Staphylococcus aureus skin and soft-tissue infections. Infect Control Hosp Epidemiol 35:1251-1256, 2014

11. Erman T, Demirhindi H, Göçer Aİ, Tuna M, İldan F, Boyar B: Risk factors for surgical site infections in neurosurgery patients with antibiotic prophylaxis. Surg Neurol 63:107113, 2005

12. Falk-Brynhildsen K, Söderquist B, Friberg O, Nilsson UG: Bacterial recolonization of the skin and wound contamination during cardiac surgery: a randomized controlled trial of the use of plastic adhesive drape compared with bare skin. J Hosp Infect 84:151-158, 2013

13. Falowski SM, Ooi YC, Bakay RA: Long-term evaluation of changes in operative technique and hardwarerelated complications with deep brain stimulation. Neuromodulation 18:670-677, 2015
14. Fenoy AJ, Simpson RK Jr: Management of device-related wound complications in deep brain stimulation surgery. $\mathbf{J}$ Neurosurg 116:1324-1332, 2012

15. Fily F, Haegelen C, Tattevin P, Buffet-Bataillon S, Revest M, Cady A, et al: Deep brain stimulation hardware-related infections: a report of 12 cases and review of the literature. Clin Infect Dis 52:1020-1023, 2011

16. Gerver S, Sinnathamby M, Bou-Antoun S, Kauser S, Canvin M, Abernethy J, et al: Epidemiological Commentary: Mandatory MRSA, MSSA and E. coli Bacteraemia and C. difficile Infection Data, 2013/14. London: Public Health England, 2014. (https://www.gov.uk/government/ uploads/system/uploads/attachment_data/file/ 330529/ HCAI_mandatory_surveillance_annual_epidemiological_ commentary_2013_14.pdf) [Accessed April 22, 2016]

17. Gould PA, Krahn AD: Complications associated with implantable cardioverter-defibrillator replacement in response to device advisories. JAMA 295:1907-1911, 2006

18. Gould PA, Gula LJ, Yee R, Skanes AC, Klein GJ, Krahn AD: Cardiovascular implantable electrophysiological device-related infections: a review. Curr Opin Cardiol 26:6-11, 2011

19. Groh GI, Groh GM: Complications rates, reoperation rates, and the learning curve in reverse shoulder arthroplasty. J Shoulder Elbow Surg 23:388-394, 2014

20. Abu Hamdeh S, Lytsy B, Ronne-Engström E: Surgical site infections in standard neurosurgery procedures - a study of incidence, impact and potential risk factors. Br J Neurosurg 28:270-275, 2014

21. Healy DG, Duignan E, Tolan M, Young VK, O'Connell B, McGovern E: Should cardiac surgery be delayed among carriers of methicillin-resistant Staphylococcus aureus to reduce methicillin-resistant Staphylococcus aureusrelated morbidity by preoperative decolonisation? Eur J Cardiothorac Surg 39:68-74, 2011

22. Kapoor R, Barnett CJ, Gutmann RM, Yildiz VO, Joseph NC, Stoicea N, et al: Preoperative prevalence of Staphylococcus aureus in cardiothoracic and neurological surgical patients. Front Public Health 2:204, 2014

23. Kim HS, Lee SG, Kim WK, Park CW, Son S: Prophylactic intrawound application of vancomycin powder in instrumented spinal fusion surgery. Korean J Spine 10:121125,2013

24. Kouyialis AT, Boviatsis EJ, Ziaka DS, Sakas DE: Use of a single semilinear incision in Deep Brain Stimulation for movement disorders. Acta Neurochir (Wien) 149:501-504, 2007

25. Kumar N, David MZ, Boyle-Vavra S, Sieth J, Daum RS: High Staphylococcus aureus colonization prevalence among patients with skin and soft tissue infections and controls in an urban emergency department. J Clin Microbiol 53:810-815, 2015

26. Lanotte M, Verna G, Panciani PP, Taveggia A, Zibetti M, Lopiano L, et al: Management of skin erosion following deep brain stimulation. Neurosurg Rev 32:111-115, 2009

27. Malchau H, Herberts P, Eisler T, Garellick G, Söderman P: The Swedish Total Hip Replacement Register. J Bone Joint Surg Am 84-A (Suppl 2):2-20, 2002 (Erratum in J Bone Joint Surg Am 86-A:363, 2004)

28. Miller JP, Acar F, Burchiel KJ: Significant reduction in stereotactic and functional neurosurgical hardware infection after local neomycin/polymyxin application. J Neurosurg 110:247-250, 2009

29. Muñoz P, Hortal J, Giannella M, Barrio JM, RodríguezCréixems M, Pérez MJ, et al: Nasal carriage of $S$. aureus increases the risk of surgical site infection after major heart surgery. J Hosp Infect 68:25-31, 2008

30. Nizet V, Ohtake T, Lauth X, Trowbridge J, Rudisill J, Dorschner RA, et al: Innate antimicrobial peptide protects the skin from invasive bacterial infection. Nature 414:454457, 2001 
31. Nuttin B, Wu H, Mayberg H, Hariz M, Gabriëls L, Galert T, et al: Consensus on guidelines for stereotactic neurosurgery for psychiatric disorders. J Neurol Neurosurg Psychiatry 85:1003-1008, 2014

32. Pepper J, Zrinzo L, Mirza B, Foltynie T, Limousin P, Hariz $\mathrm{M}$ : The risk of hardware infection in deep brain stimulation surgery is greater at impulse generator replacement than at the primary procedure. Stereotact Funct Neurosurg 91:5665, 2013

33. Pollock AV, Leaper DJ, Evans M: Single dose intra-incisional antibiotic prophylaxis of surgical wound sepsis: a controlled trial of cephaloridine and ampicillin. Br J Surg 64:322-325, 1977

34. Ragel BT, Browd SR, Schmidt RH: Surgical shunt infection: significant reduction when using intraventricular and systemic antibiotic agents. J Neurosurg 105:242-247, 2006

35. Scher KS, Peoples JB: Combined use of topical and systemic antibiotics. Am J Surg 161:422-425, 1991

36. Sillay KA, Larson PS, Starr PA: Deep brain stimulator hardware-related infections: incidence and management in a large series. Neurosurgery 62:360-367, 2008

37. Solmaz B, Tatarli N, Ceylan D, Bayri Y, Ziyal Mİ, Şeker A: A sine-wave-shaped skin incision for inserting deep-brain stimulators. Acta Neurochir (Wien) 156:1523-1525, 2014

38. Thrane JF, Sunde NA, Bergholt B, Rosendal F: Increasing infection rate in multiple implanted pulse generator changes in movement disorder patients treated with deep brain stimulation. Stereotact Funct Neurosurg 92:360-364, 2014

39. Tolleson C, Stroh J, Ehrenfeld J, Neimat J, Konrad P, Phibbs F: The factors involved in deep brain stimulation infection: a large case series. Stereotact Funct Neurosurg 92:227-233, 2014

40. Tong F, Ramirez-Zamora A, Gee L, Pilitsis J: Unusual complications of deep brain stimulation. Neurosurg Rev 38:245-252, 2015

41. Voges J, Waerzeggers Y, Maarouf M, Lehrke R, Koulousakis A, Lenartz D, et al: Deep-brain stimulation: long-term analysis of complications caused by hardware and surgeryexperiences from a single centre. J Neurol Neurosurg Psychiatry 77:868-872, 2006

42. Volkmann J, Allert N, Voges J, Weiss PH, Freund HJ, Sturm $\mathrm{V}$ : Safety and efficacy of pallidal or subthalamic nucleus stimulation in advanced PD. Neurology 56:548-551, 2001

43. Wilcox M, Cowling P, Duerden B, Fry C, Hopkins S, Jenks $P$, et al: Implementation of Modified Admission MRSA Screening Guidance for NHS 2014. London: Department of Health, 2014 (https://www.gov.uk/government/ uploads/system/uploads/attachment_data/file/345144/ Implementation_of_modified_admission_MRSA_screening guidance_for_NHS.pdf) [Accessed April 22, 2016]
44. Yano K, Minoda Y, Sakawa A, Kuwano Y, Kondo K, Fukushima W, et al: Positive nasal culture of methicillinresistant Staphylococcus aureus (MRSA) is a risk factor for surgical site infection in orthopedics. Acta Orthop 80:486490, 2009

45. Yaw LK, Robinson JO, Ho KM: A comparison of long-term outcomes after meticillin-resistant and meticillin-sensitive Staphylococcus aureus bacteraemia: an observational cohort study. Lancet Infect Dis 14:967-975, 2014

46. Zrinzo L, Foltynie T, Limousin P, Hariz MI: Reducing hemorrhagic complications in functional neurosurgery: a large case series and systematic literature review. J Neurosurg 116:84-94, 2012

\section{Disclosures}

Dr. Hyam has received honoraria from Medtronic and St. Jude Medical to attend academic conferences. Dr. Limousin has received honoraria from Medtronic, St. Jude, and Boston Scientific for lectures and for expenses for conferences. Dr. Hariz has received honoraria from Medtronic for travel expenses for speaking at meetings. Dr. Zrinzo has received honoraria from Medtronic and St. Jude Medical for educational talks at meetings.

\section{Author Contributions}

Conception and design: Zrinzo, Pepper, Meliak, Hariz. Acquisition of data: Zrinzo, Pepper, Milabo, Candelario. Analysis and interpretation of data: Zrinzo, Pepper, Foltynie, Curtis. Drafting the article: Pepper, Hariz. Critically revising the article: Zrinzo, Pepper, Akram, Hyam, Foltynie, Limousin, Curtis, Hariz. Reviewed submitted version of manuscript: Zrinzo, Pepper, Akram, Hyam, Foltynie, Limousin, Curtis, Hariz. Approved the final version of the manuscript on behalf of all authors: Zrinzo. Statistical analysis: Zrinzo, Pepper. Administrative/technical/ material support: Pepper. Study supervision: Zrinzo, Hariz.

\section{Supplemental Information Previous Presentations}

An abstract (oral presentation) of this work was previously presented at the biennial congress of the World Society for Stereotactic and Functional Neurosurgery in September 2015 in Mumbai, India, and at the Society of British Neurological Surgeons Meeting in September 2015 in York, United Kingdom.

\section{Correspondence}

Ludvic Zrinzo, Unit of Functional Neurosurgery, UCL Institute of Neurology, Queen Square, London WC1N 3BG, United Kingdom.email: 1.zrinzo@ucl.ac.uk. 